\title{
An indirect method for the oxidation of aryl phosphites to phosphates and aryl selenoxides to selenones
}

\author{
Nikoletta Sofikiti and Manolis Stratakis* \\ Department of Chemistry, University of Crete, Iraklion 71409, Greece \\ E-mail: stratakis@chemistry.uoc.gr
}
Dedicated to my first teacher of organic chemistry, Professor Anastasios Varvoglis, on the occasion of his $65^{\text {th }}$ birthdate
(received 03 Feb 03; accepted 06 Mar 03; published on the web 18 Mar 03)

\begin{abstract}
Aryl phosphites and aryl selenoxides are unreactive towards singlet oxygen. However, in the dye-sensitized co-photooxygenation of 1 equivalent of an aryl phosphite or an aryl selenoxide with 1.2-1.5 equivalents of dimethyl sulfide, the corresponding aryl phosphates and aryl selenones are produced with $>90 \%$ isolated yield (10 examples). The reaction is applicable to phosphites and selenoxides with either electron-donating or electron-withdrawing substituents on the aryl ring.
\end{abstract}

Keywords: Oxidation, singlet oxygen, sulfides, selenoxides, phosphites

\section{Introduction}

Sulfides react smoothly with singlet oxygen $\left({ }^{1} \mathrm{O}_{2}\right)$ to produce mainly sulfoxides. ${ }^{1}$ The mechanism of the reaction has been extensively studied by several research groups ${ }^{2}$ in the past and continues to be an active topic of singlet oxygen chemistry. For the photooxygenation of sulfides in aprotic solvents, mainly two intermediates have been proposed, the persulfoxide ${ }^{3} \mathbf{1}$, and the hydroperoxy sulfonium ylide 2 . On the other hand, in protic solvents such as methanol, the existence of the sulfurane intermediate 3 has been postulated (Scheme 1). 


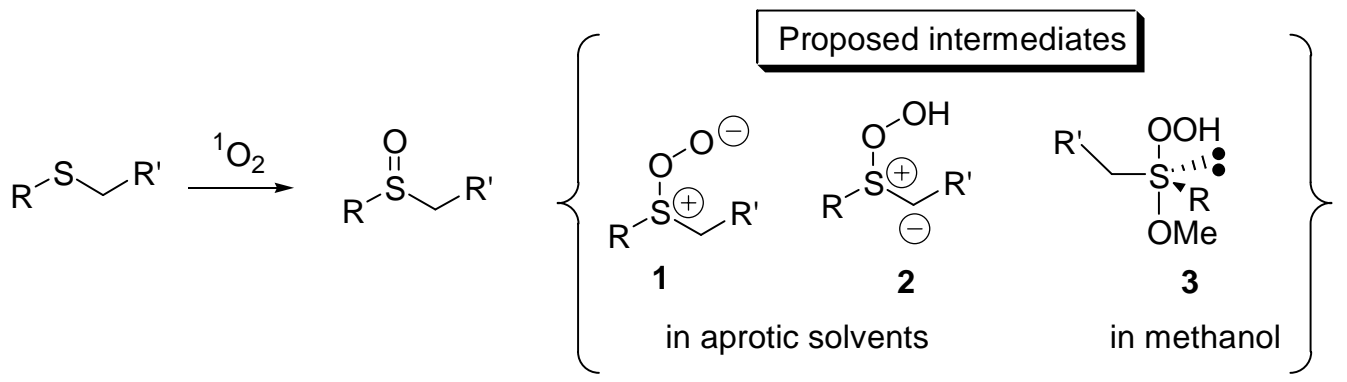

Scheme 1. Proposed intermediates in the photooxygenation of sulfides.

The peroxy intermediates in the photooxygenation of alkyl sulfides in aprotic solvents have been trapped by trimethyl phosphite, ${ }^{4}$ sulfonamides, ${ }^{5}$ and less efficiently by aryl sulfoxides ${ }^{6}$ (Scheme 2). Later studies by Clennan and co-workers ${ }^{4}$ revealed that triphenyl phosphite is a better trapping reagent compared to trimethyl phosphite, and around 20 times more efficient than diphenyl sulfoxide. The trapping reaction is less favourable in methanol.

$$
\begin{aligned}
& \mathrm{Et}^{-}{ }^{-\mathrm{S}}{ }_{\mathrm{Et}} \underset{(\mathrm{MeO})_{3} \mathrm{P}}{\stackrel{{ }^{1} \mathrm{O}_{2}}{\longrightarrow}} \mathrm{Et}^{-} \stackrel{\stackrel{\mathrm{S}}{\mathrm{S}}{ }_{\mathrm{Et}}}{\underset{\mathrm{N}}{\longrightarrow}}+(\mathrm{MeO})_{3} \mathrm{PO} \quad \mathrm{Et}_{2} \mathrm{~S} /(\mathrm{MeO})_{3} \mathrm{P} \sim 1 / 5
\end{aligned}
$$

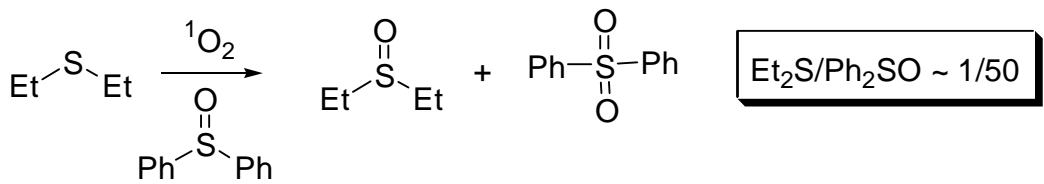

Scheme 2. Photooxygenation of diethyl sulfide in the presence of trimethyl phosphite or diphenyl sulfoxide.

In addition, recent studies from our research group ${ }^{7}$ have shown that although aryl selenoxides are completely inert with ${ }^{1} \mathrm{O}_{2}$, they can trap the peroxy intermediates in the photooxygenation of dimethyl sulfide much more efficiently than the sulfur-analogues, diaryl sulfoxides, accomplish. For example, in the co-oxidation of one equivalent of diphenyl selenoxide with 1.2-1.3 equivalents of dimethyl sulfide, the selenoxide is oxidized quantitatively to diphenyl selenone (Scheme 3). The mechanistic details for this trapping reaction are currently under investigation, by means of varying the electron density at the aryl rings of the selenoxides.

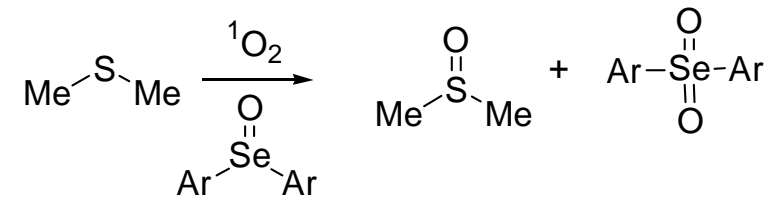

Scheme 3. Trapping of oxygenated intermediates in the photooxygenation of sulfides by aryl selenoxides. 
We sought to apply these co-photooxygenation trapping reactions for synthetic purposes. Hence, the most efficient trapping reagents, triaryl phosphites and diaryl selenoxides were chosen, to perform their indirect oxidation to phosphates and selenones respectively. Dimethyl sulfide was used because its oxidation product, dimethyl sulfoxide, is water missible and can be easily removed by extraction of the crude photooxygenation mixture.

\section{Results and Discussion}

The synthesis of triaryl phosphites and diaryl selenoxides was accomplished as shown in the following Scheme 4, using known literature procedures.

$$
\begin{aligned}
& \text { ArOH } \underset{\mathrm{Et}_{2} \mathrm{O} / \mathrm{Et}_{3} \mathrm{~N}}{\stackrel{\mathrm{PBr}_{3}}{\longrightarrow}}(\mathrm{ArO})_{3} \mathrm{P} \\
& \mathrm{ArN}_{2}{ }^{+} \mathrm{BF}_{4}^{-} \stackrel{\text { NaHSe }}{\longrightarrow} \mathrm{Ar}^{-}{ }_{-}^{-\mathrm{Se}_{-}} \stackrel{\mathrm{NCS}}{\mathrm{OH}^{-}} \mathrm{Ar}^{-}{ }^{\text {SI }}{ }_{-\mathrm{Ar}}
\end{aligned}
$$

Scheme 4. Synthesis of aryl phosphites and aryl selenoxides.

A variety of aryl phosphites and aryl selenoxides possessing either electron-donating or electron-withdrawing substituents on the aryl ring were quantitatively oxidized if cophotooxygenated with 1.2-1.5 equivalents of dimethyl sulfide. The results are presented in Tables 1 and 2.

Table 1. Oxidation of aryl phosphites to phosphates

$$
(\mathrm{ArO})_{3} \mathrm{P} \stackrel{[\mathrm{O}]}{\longrightarrow}(\mathrm{ArO})_{3} \mathrm{PO}
$$

\begin{tabular}{ccccc}
\hline Entry & Ar & $\begin{array}{c}\text { Equiv. of } \mathrm{Me}_{2} \mathrm{~S} \text { per } \\
\text { equiv. of }(\mathrm{ArO})_{3} \mathrm{P}\end{array}$ & $\begin{array}{c}\text { Reaction time } \\
(\mathrm{min})\end{array}$ & Yield (\%) \\
\hline 1 & Phenyl & 1.5 & 15 & $93^{\mathrm{a}}$ \\
2 & p-Tolyl & 1.5 & 5 & $>95^{\mathrm{b}}$ \\
3 & p-Methoxyphenyl & 1.5 & 5 & $>95^{\mathrm{b}}$ \\
4 & p-Chlorophenyl & 1.5 & 5 & $>95^{\mathrm{b}}$ \\
5 & p-Iodophenyl & 1.5 & 15 & $92^{\mathrm{a}}$ \\
6 & p-Fluorophenyl & 1.5 & 5 & $>95^{\mathrm{b}}$ \\
7 & m-Chlorophenyl & 1.5 & 5 & $>95^{\mathrm{b}}$ \\
\hline
\end{tabular}

${ }^{\mathrm{a}}$ Isolated yield. ${ }^{\mathrm{b}}$ Yield by ${ }^{1} \mathrm{H}$ NMR. 
Table 2. Oxidation of selenoxides to selenones

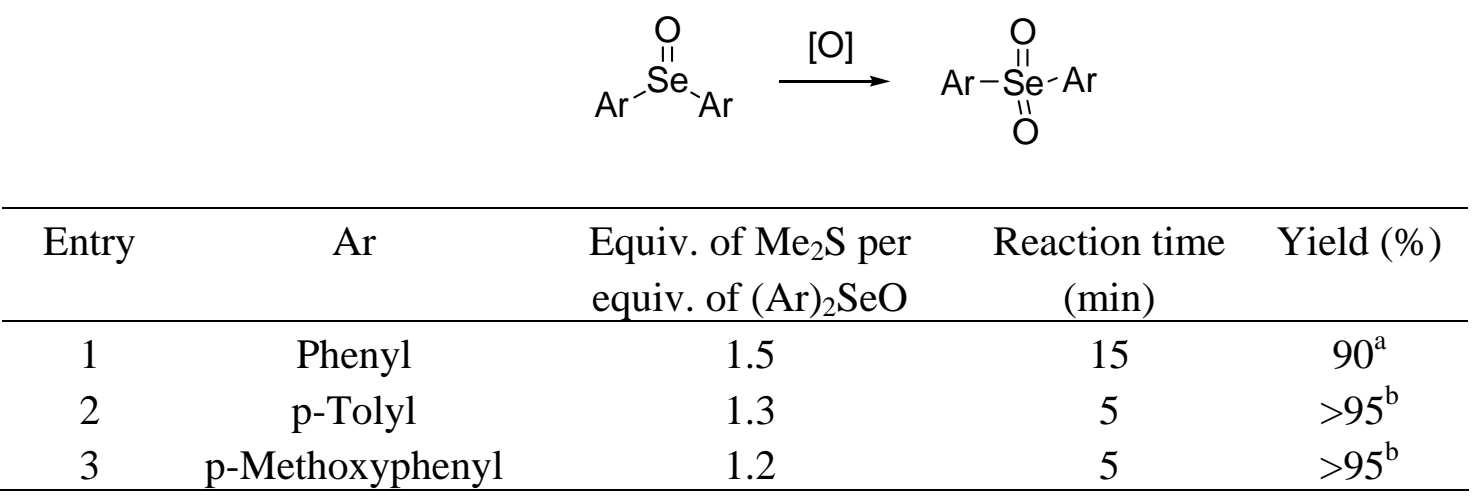

${ }^{\mathrm{a}}$ Isolated yield. ${ }^{\mathrm{b}}$ Yield by ${ }^{1} \mathrm{H}$ NMR.

The majority of the experiments were performed in NMR scale ( 10 mg of phosphite or selenoxide), apart of entries 1 and 5 in Table 1, and entry 1 in Table 2, which were performed on a $500 \mathrm{mg}$ scale.

It is remarkable that although phosphites and selenoxides are unreactive with singlet oxygen, they are quantitatively oxidized under the reaction conditions. The oxidant is more likely one of the postulated peroxy intermediates $\mathbf{1}$ or $\mathbf{2}$ shown in Scheme 1. This indirect oxidation protocol is novel, highly efficient and applicable to a variety of phosphites and selenoxides, bearing either electron-donating or electron-withdrawing groups.

\section{Experimental Section}

General Procedures. Nuclear magnetic resonance spectra were recorded on a $500 \mathrm{MHz}$ spectrometer, in $\mathrm{CDCl}_{3}$. Isomeric purities were determined by ${ }^{1} \mathrm{H} \mathrm{NMR}$ and ${ }^{31} \mathrm{P} \mathrm{NMR}$ spectroscopy and by analytical gas chromatography on a 60 m HP-5 capillary column.

Preparation of aryl phosphates. Triphenyl phosphite is commercially available. The rest of the phosphites were prepared ${ }^{8}$ as follows. In a flame dried flask containing $40 \mathrm{~mL}$ of anhydrous ether, 40 mmoles of triethyl amine and 33 mmoles of the phenol were added dropwise at $0{ }^{\circ} \mathrm{C}, 10$ mmoles of $\mathrm{PBr}_{3}$. After $30 \mathrm{~min}$, the reaction mixture was filtered, extracted with $1 \mathrm{~N} \mathrm{HCl}$, saturated solution of $\mathrm{NaHCO}_{3}$ and finally with brine. Removal of the solvent, afforded the phosphites in $50-80 \%$ yield.

${ }^{1} \mathrm{H}$ NMR data of the aryl phosphites. p-Methylphenyl: $7.12(\mathrm{~d}, \mathrm{~J}=8.0 \mathrm{~Hz}, 6 \mathrm{H}), 7.04(\mathrm{~d}, \mathrm{~J}=$ $8.0 \mathrm{~Hz}, 6 \mathrm{H}$ ), 2.33 (s, 9H); p-Methoxyphenyl: 7.06 (d, J = 9.0 Hz, 6H), 6.84 (d, J = 9.0 Hz, 6H), 3.79 (s, 9H); p-Chlorophenyl: 7.30 (d, J = $8.5 \mathrm{~Hz}, 6 \mathrm{H}), 7.06$ (d, J = 8.5 Hz, 6H); p-Iodophenyl: 
7.63 (d, J = 8.5 Hz, 6H), 6.87 (d, J = 8.5 Hz, 6H); m-Chlorophenyl: 7.27 (t, J = 8.0 Hz, 3H), 7.16 (d, J = 8.0 Hz, 3H), 7.15 (s, 3H), 7.03 (d, J = 8.0 Hz, 3H); p-Fluorophenyl: 7.00-7.09 (m, 12H).

${ }^{31} \mathrm{P}$ NMR data of the aryl phosphites. $p$-Methylphenyl: 129.18; $p$-Methoxyphenyl: 129.55; $p$ Chlorophenyl: 127.55; p-Iodophenyl: 127.16; m-Chlorophenyl: 127.23; p-Fluorophenyl: 128.20.

Preparation of aryl selenoxides. Apart of bis(p-methoxyphenyl) selenoxide which is comm.erciallly available by TCI, diphenyl selenoxide and bis(p-tolyl) selenoxide ${ }^{9}$ were prepared $^{10}$ by oxidation of the corresponding selenides with $N$-chlorosucinimide followed by alkaline hydrolysis. Diphenyl selenide is commercially available, while bis(p-tolyl) selenide ${ }^{11}$ was prepared ${ }^{12}$ by coupling of the diazonium $\mathrm{BF}_{4}{ }^{-}$salt of $p$-anisidine with NaHSe, produced in situ by the reaction of $\mathrm{NaBH}_{4}$ with Se metal. ${ }^{1} \mathrm{H}$ NMR of bis(p-tolyl) selenide: 7.36 (d, J = 8.0 Hz, 4H), 7.08 (d, J = 8.0 Hz, 4H), $2.33(\mathrm{~s}, 6 \mathrm{H}) .{ }^{1} \mathrm{H}$ NMR data of the aryl selenoxides. Diphenyl selenoxide: $7.71(\mathrm{~m}, 4 \mathrm{H}), 7.47$ (m, 6H); Bis(p-tolyl) selenoxide: 7.56 (d, J = 8.0 Hz, 4H), 7.27 (d, $\mathrm{J}=8.0 \mathrm{~Hz}, 4 \mathrm{H}), 2.37$ (s, 6H).

\section{General procedure for the photooxygenation of dimethyl sulfide in the presence of phosphite or selenoxide}

In a test tube containing $510 \mathrm{mg}$ of triphenyl phosphite were added $20 \mathrm{~mL}$ of dichloromethane, $150 \mu \mathrm{L}$ of dimethyl sulfide and $1 \mathrm{mg}$ of methylene blue. The tube was irradiated with a $300 \mathrm{~W}$ Xenon lamp at $0{ }^{\circ} \mathrm{C}$, for $15 \mathrm{~min}$, under a constant flow of oxygen. The solvent was removed under vacuum, and subsequently the residue was diluted with ether and extracted with water. Removal of the ether under reduced pressure afforded $510 \mathrm{mg}$ of triphenyl phosphate in $>97 \%$ purity (93\% yield). ${ }^{1} \mathrm{H}$ NMR data of the aryl phosphates. p-Methylphenyl: 7.05 (d, J = $8.5 \mathrm{~Hz}$, 6H), 7.01 (d, J = 8.5 Hz, 6H), 2.91 (s, 9H); p-Methoxyphenyl: 7.14 (d, J = 8.0 Hz, 6H), 6.84 (d, J $=8.0 \mathrm{~Hz}, 6 \mathrm{H}), 3.78$ (s, 9H); p-Chlorophenyl: 7.33 (d, $J=8.5 \mathrm{~Hz}, 6 \mathrm{H}), 7.16$ (d, $J=8.5 \mathrm{~Hz}, 6 \mathrm{H})$; $p$ Iodophenyl: 7.58 (d, J = 8.5 Hz, 6H), 6.98 (d, $J=8.5 \mathrm{~Hz}, 6 \mathrm{H}) ; m$-Chlorophenyl: 7.25 (t, J = 8.0 Hz, 3H), 7.18 (s, 3H), 7.17 (d, $J=8.0 \mathrm{~Hz}, 3 \mathrm{H}), 7.08$ (d, $J=8.0 \mathrm{~Hz}, 3 \mathrm{H}) ;$-Fluorophenyl: 7.14 (dd, $\left.\mathrm{J}_{1}=8.5 \mathrm{~Hz}, J_{2}=4.5 \mathrm{~Hz}, 6 \mathrm{H}\right), 7.00\left(\mathrm{dd}, J_{1}=8.5 \mathrm{~Hz}, \mathrm{~J}_{2}=8.5 \mathrm{~Hz}, 6 \mathrm{H}\right) .{ }^{31} \mathrm{P}$ NMR data of the aryl phosphates. $p$-Methylphenyl: -16.33; p-Methoxyphenyl: -15.33; p-Chlorophenyl: -17.18; $p$ Iodophenyl: -17.66; $m$-Chlorophenyl: -17.85; $p$-Fluorophenyl: -16.31.

${ }^{1} \mathrm{H}$ NMR data of the aryl selenones. Diphenyl selenone: 8.00 (d, $\left.J=7.5 \mathrm{~Hz}, 4 \mathrm{H}\right), 7.68(\mathrm{t}, \mathrm{J}=7.5$ $\mathrm{Hz}, 2 \mathrm{H}), 7.61$ (m, 4H); Bis(p-tolyl) selenone: 7.84 (d, $J=8.5 \mathrm{~Hz}, 4 \mathrm{H}), 7.38$ (d, J = $8.5 \mathrm{~Hz}, 4 \mathrm{H})$, 2.97 (s, 6H); Bis(p-methoxyphenyl) selenone: 7.90 (d, $J=9.0 \mathrm{~Hz}, 4 \mathrm{H}), 7.05$ (d, J = 9.0 Hz, 4H), $3.87(\mathrm{~s}, 6 \mathrm{H})$.

\section{Acknowledgments}

We thank the Greek Ministry of Education (B' EPEAEK Graduate Program) for financial support. 


\section{References}

1. Frimer, A. A.; Stephenson, L. M. In Singlet Oxygen. Reactions, Modes and Products, A.A. Frimer Eds. CRC Press: Boca Raton, FL 1985.

2. Clennan, E. L. Acc. Chem. Res. 2001, 34, 875.

3. Liang, J.-J.; Gu, C. L.; Kacher, M. L.; Foote C. S. J. Am. Chem. Soc. 1983, 105, 4717.

4. Nahm, K.; Foote, C. S. J. Am. Chem. Soc. 1989, 111, 1909.

5. Sawaki, Y.; Ogata, Y. J. Am. Chem. Soc. 1981, 103, 5947.

6. Clennan, E. L.; Stensaas, K. L.; Rupert, S. D. Heteromat. Chem. 1998, 9, 51.

7. Sofikiti, N.; Rabalakos, C.; Stratakis, M. Unpublished results.

8. Stratakis, M.; Rabalakos, C.; Sofikiti, N. Tetrahedron Lett. 2003, 44, 349.

9. Furukawa, N.; Ogawa, S.; Matsumura, K.; Fujihara, H. J. Org. Chem. 1991, 56, 6341.

10. Detty, M. R. J. Org. Chem. 1980, 45, 274.

11. Watanabe, S.; Yamamoto, K.; Itagaki, K.; Iwamura, T.; Iwama, T.; Kataoka, T. Tetrahedron 2000, 56, 855. We thank professor T. Kataoka for providing us with detailed spectral data of bis(p-tolyl) selenide.

12. Chen, C.; Qiu, M.; Zhou, X. J. Synth. Commun. 1991, 21, 1729. 